doi:10.1136/gut.2010.233445

Predictors of failure of pneumatic dilatation in Achalasia

Most gastroenterologists prefer pneumatic dilatation (PD) as first-line therapy for Achalasia but the reported success rates of this procedure vary widely. In this large study from The Netherlands, Alderliesten et al reviewed 336 patients who were treated with PD between 1974 and 2006. The median follow-up was 129 months (range 1-378). Relatively few complications related to PD occurred (listed in table 1) and there were no fatalities. 111 patients had symptom recurrence requiring repeated treatment. Symptoms recurred after a mean follow-up of 51 months (range 1-348). The authors found that young age at presentation, a manometric pattern of classic achalasia, high LOS pressure 3 months after $\mathrm{PD}$, and incomplete obliteration of the balloon's waist during $\mathrm{PD}$ were the most important predicting factors for the need for repeated treatment during follow-up. Patients who meet one or more of these characteristics may be considered earlier for alternative treatment, such as surgery. See page 10.

Table 1 Complications related PD

\begin{tabular}{lll}
\hline Complication & $\begin{array}{l}\text { No of } \\
\text { procedures } \\
\text { (n=985) }\end{array}$ & $\begin{array}{l}\text { Percentage } \\
(\%)\end{array}$ \\
\hline None & 945 & 95.9 \\
Post-procedural pain & 31 & 3.1 \\
Fever & 16 & 1.6 \\
Perforation & 13 & 1.3 \\
Aspiration & 3 & 0.3 \\
Bleeding & 2 & 0.2 \\
\hline
\end{tabular}

\section{Confocal laser endomicroscopy} and intramucosal bacteria in IBD Enteric bacteria play an important role in the pathogenesis of IBD. Confocal laser endomicroscopy (CLE) is the only technology that enables microscopic imaging within the mucosal layer of the gut at subcellular resolution. In this landmark study, Moussata et al developed a fluorescein-aided CLE method to visualise intramucosal enteric bacteria in vivo and determined the involved mucosal area in the colon and ileum in patients with ulcerative colitis (UC) and Crohn's disease (CD). Using FISH as the gold standard,

Emad El-Omar, Severine Vermeire and Alexander Gerbes, Editor and Deputy Editors

CLE had a sensitivity of $89 \%$ and specificity of $100 \%$ to identify intramucosal bacteria in patients. In a retrospective study, 113 patients with CD and UC had intramucosal bacteria significantly more often than 50 control patients $(66 \%$ vs $60 \%$ vs $14 \%, p<0.001)$. This result was confirmed in a prospective study in which 10 patients with CD and 10 with UC had a significantly wider distribution of involvement with intramucosal bacteria in the colon and terminal ileum compared with 10 controls $(85.2 \%$ vs $75.9 \%$ vs $16.8 \%, \mathrm{p}<0.0001$ ) (see figure below). This novel endoscopic technique could be a valuable tool in the elucidation of the pathogenesis of IBD and the development of new clinical algorithms. See page $\mathbf{2 6}$.

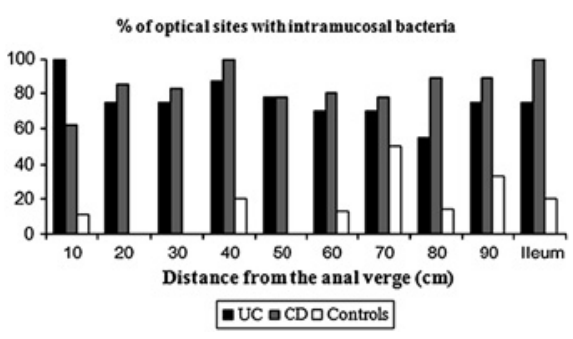

Increased intramucosal bacteria in patients with inflammatory bowel disease.

\section{Dietary sphingomyelin induces apoptosis}

The majority of inflammatory bowel disease patients have intestinal permeability defects because of increased rates of apoptosis among intestinal epithelial cells (IEC). Sphingomyelin is a constituent in food derived from animals and digestion of sphingomyelin in the small intestine or colon results in ceramide, which in turn induces the maturation of procathepsin D into the mature enzyme cathepsin, a novel mediator of apoptosis. In this study in acute DSS colitic C57-BL/6 mice, the authors administered 4 or $8 \mathrm{mg}$ sphingomyelin/day by oral gavage and isolated IEC to study apoptosis. The results (PI, TUNEL staining, immunohistochemistry and Western Blot) showed increased apoptosis of IEC under dietary sphingomyelin. The authors confirmed their results in the IL- $10^{-/-}$mouse model where aggravation of mucosal inflammation was also observed. They conclude that apoptosis of IEC induced by dietary sphingomyelin may shorten the physiological life cycle of IEC and impair barrier function of the intestinal mucosa. Dietary sphingomyelin may increase intestinal inflammation. See page 55

Antibiotics use and inflammatory bowel diseases in childhood

Intestinal dysbiosis has been described in inflammatory bowel diseases (IBD), pointing towards a role of the gut microbiota in the development of disease. As antibiotics alter the composition of the intestinal microbiota, the authors in this study evaluated if an association exists between antibiotics use in childhood and the development of IBD. They conducted a nationwide cohort study of all Danish children born between 1995 to 2003 $(\mathrm{N}=577627)$ from whom they had
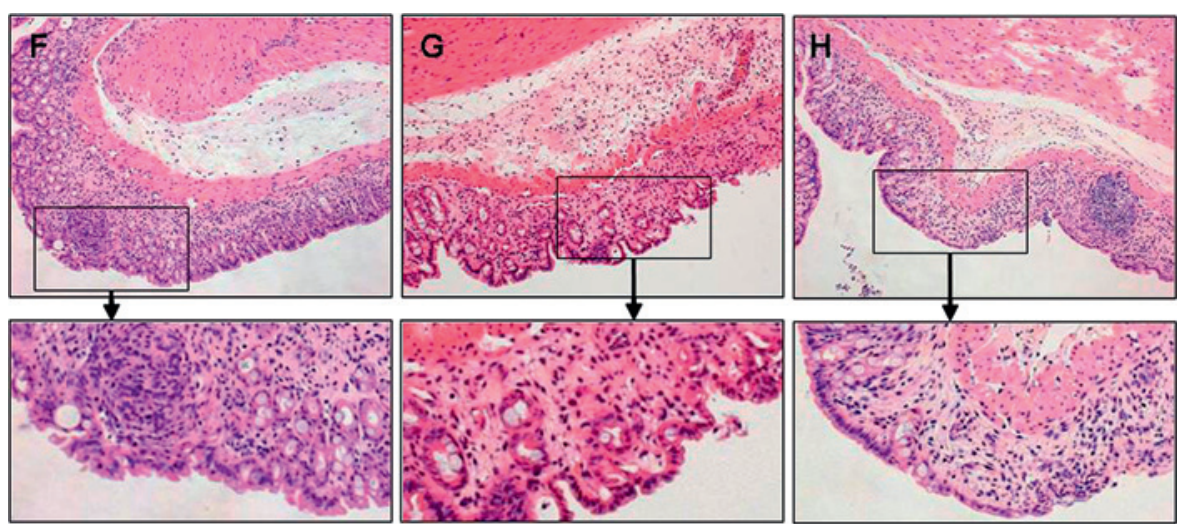

H\&E staining of the colon after DSS colitis (F) DSS/water, (G) DSS/4 mg sphingomyelin, (H) DSS/ $8 \mathrm{mg}$ sphingomyelin. 
information on filled antibiotics prescriptions, on the development of IBD and other potential confounding variables. IBD was diagnosed in 117 children. The $\mathrm{RR}$ of developing IBD was 1.84 (95\% CI 1.08 to 3.15 ) comparing antibiotics users to non-users. This association was true only for Crohn's disease (RR 3.41) and was strongest in the first 3 months following antibiotics use (RR 4.43), and among children with 7 or more courses of antibiotics (RR 7.32). The authors conclude that antibiotics use in childhood may be a risk factor for development of IBD later in life. See page 49.

\section{HBV viraemia-the burden of heritage}

Hepatitis B virus carriers exhibit a wide variation of blood HBV DNA levels and

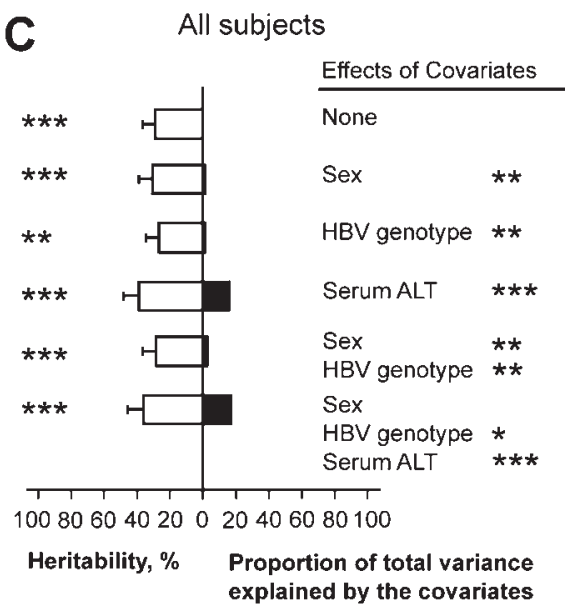

D Subjects with normal ALT

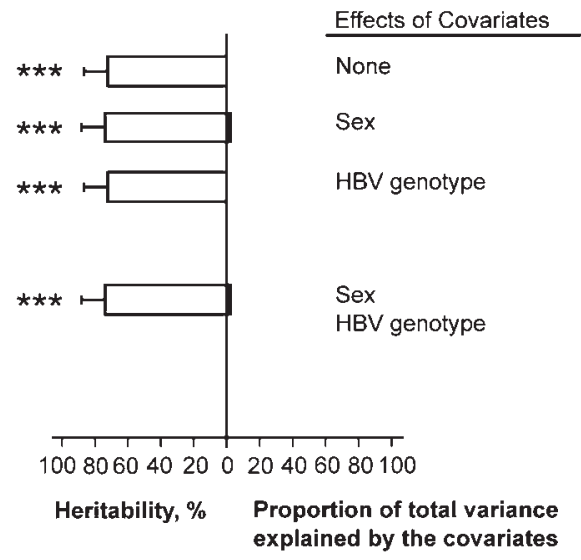

A novel technique to reduce liver fibrosis

Natural killer (NK) cells inhibit liver fibrosis by killing hepatic stellate cells (HSC). The present study introduces a novel technique to activate NK cells by silencing inhibitory receptors on their surface. By use of small interfering RNA NK cell activity was stimulated and HSC activity was attenuated in co-culture. In an animal model fibrosis of the liver was clearly reduced (see figures below). This immunomodulatory strategy may represent a novel antifibrotic treatment. See page 90. with levels and persistence of HBV viraemia (see figures below). Now these novel and interesting findings should be examined in other populations with less intrafamilial and perinatal HBV transmission. See page 99.
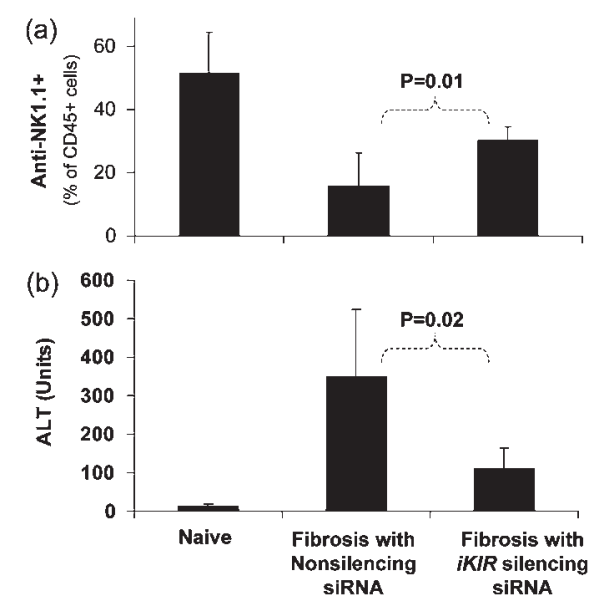

siRNA increases NK cell content and reduces $A L T$ in vivo.

Heritability estimates. 